Pure and Applied Mathematics Quarterly

Volume 4, Number 1

(Special Issue: In honor of

Gregory Margulis, Part 2 of 2)

$181-202,2008$

\title{
A Measurable Cartan Theorem and Applications to Deformation Rigidity in Complex Hyperbolic Geometry
}

\author{
Marc Burger and Alessandra Iozzi \\ To Grisha, who keeps inspiring so many of us
}

\begin{abstract}
We establish a version for measurable maps of a theorem of E. Cartan [10] according to which a bijection of the boundary of complex hyperbolic plane mapping chains into chains comes from an isometry. As an application, we prove a global rigidity result which was originally announced in [5] and [18] with a sketch of a proof using bounded cohomology techniques and then proven by Koziarz and Maubon in [19] using harmonic map techniques. As a corollary one obtains that a lattice in $\mathrm{SU}(p, 1)$ cannot be deformed nontrivially in $\mathrm{SU}(q, 1), q \geq p$, if either $p \geq 2$ or the lattice is cocompact. This generalizes to noncocompact lattices a theorem of Goldman and Millson, [14].
\end{abstract}

\section{INTRODUCTION}

The ideal boundary $\partial \mathcal{H}_{\mathbb{C}}^{\ell}$ of complex hyperbolic $\ell$-space $\mathcal{H}_{\mathbb{C}}^{\ell}$ carries a rich geometry whose "lines" are the chains. A chain in $\partial \mathcal{H}_{\mathbb{C}}^{\ell}$ is by definition the boundary of a complex geodesic in $\mathcal{H}_{\mathbb{C}}^{\ell}$; as such it is a circle equipped with a canonical orientation, and it is uniquely determined by any two points lying on it. The "geometry of chains" was first studied by E. Cartan who showed that, analogously to the Fundamental Theorem of Projective Geometry [1, Theorem 2.26], any automorphism of the incidence graph of the geometry of chains comes, for $\ell \geq 2$, from an isometry of $\mathcal{H}_{\mathbb{C}}^{\ell},[10]$. The main result of this paper is a consequence of an analog of Cartan's theorem (see Theorem 2.1) in the measurable setting. Namely we prove the following:

Received January 25, 2007.

A.I. was partially supported by FNS grant PP002-102765. 
TheOREM 1. Let $p \geq 2$ and let $\varphi: \partial \mathcal{H}_{\mathbb{C}}^{p} \rightarrow \partial \mathcal{H}_{\mathbb{C}}^{q}$ be a measurable map such that for almost every chain and almost every triple $(\xi, \eta, \zeta)$ of distinct points on it, the triple $\varphi(\xi), \varphi(\eta), \varphi(\zeta)$ consists also of distinct points on a chain and has the same orientation as $(\xi, \eta, \zeta)$. Then either

(1) there is a chain $C \subset \partial \mathcal{H}_{\mathbb{C}}^{q}$ such that $\varphi(\xi) \in C$ for almost every $\xi \in \partial \mathcal{H}_{\mathbb{C}}^{p}$, or

(2) there is an isometric holomorphic embedding $F: \mathcal{H}_{\mathbb{C}}^{p} \rightarrow \mathcal{H}_{\mathbb{C}}^{q}$ such that the map

$$
\partial F: \partial \mathcal{H}_{\mathbb{C}}^{p} \rightarrow \partial \mathcal{H}_{\mathbb{C}}^{q}
$$

induced on the boundary coincides with $\varphi$ almost everywhere.

The precise meaning of the first "almost everywhere" condition in the above theorem comes from the fact that the space of chains in $\partial \mathcal{H}_{\mathbb{C}}^{p}$ is a homogeneous space for $\mathrm{SU}(p, 1)$ and, as such, carries a canonical invariant measure class.

As a corollary of this result and of the results in [3] and [4], we obtain a rigidity theorem for representations of lattices $\Gamma$ in $\mathrm{SU}(p, 1)$ into $\mathrm{PU}(q, 1)$. To describe this, let us recall the invariant $i_{\rho}$, first introduced in $[5,18]$, associated to any such homomorphism $\rho: \Gamma \rightarrow \mathrm{PU}(q, 1)$. Let $\mathcal{H}_{\mathbb{C}}^{q}$ be the complex hyperbolic $q$-space endowed with the Riemannian metric of constant holomorphic sectional curvature -1 , and let $\omega_{q}$ be the corresponding Kähler form. Let $\kappa_{q} \in \mathrm{H}_{\mathrm{c}}^{2}(\mathrm{PU}(q, 1), \mathbb{R})$ be the continuous class which corresponds to $\omega_{q}$ via the van Est isomorphism [21]. Let $M:=\Gamma \backslash \mathcal{H}_{\mathbb{C}}^{p}$ be the finite volume quotient and assume that either $p \geq 2$ or $M$ is compact. Then the $\mathrm{E}^{2}$-cohomology space $\mathrm{H}_{(2)}^{2}(M)$ injects into $\mathrm{H}_{\mathrm{dR}}^{2}(M) \simeq \mathrm{H}^{2}(\Gamma, \mathbb{R})$, and it is a fact that the pullback $\rho^{(2)}\left(\kappa_{q}\right)$, seen as an element of $\mathrm{H}_{\mathrm{dR}}^{2}(M)$, belongs to the subspace $\mathrm{H}_{(2)}^{2}(M)$, see [3].

Since $M$ is at any rate locally Hermitian symmetric, we may identify $\mathrm{H}_{(2)}^{k}(M)$ with the space of harmonic $k$-forms which are $\mathrm{E}^{2}$ on $M$; in particular $\mathrm{H}_{(2)}^{2}(M)$ comes with the natural inner product of $\mathrm{E}^{2}$-forms, and the Kähler form $\omega_{M}$ coming from $\omega_{p}$ defines then an element of $\mathrm{H}_{(2)}^{2}(M)$. Our invariant $i_{\rho}$ associated to $\rho$ is then defined by:

$$
i_{\rho}:=\frac{\left\langle\rho^{(2)}\left(\kappa_{q}\right), \omega_{M}\right\rangle}{\left\langle\omega_{M}, \omega_{M}\right\rangle}
$$

and, with the above normalization of hyperbolic metric, we have

$$
\left|i_{\rho}\right| \leq 1 \text {. }
$$

Let us call a representation $\rho: \Gamma \rightarrow \mathrm{PU}(q, 1)$ maximal if $i_{\rho}=1$. We observe that the study of representations with $i_{\rho}=-1$ reduces to the study of maximal representations by conjugating $\rho$ with an antiholomorphic isometry of $\mathcal{H}_{\mathbb{C}}^{q}$. 
Theorem 2. Let $\Gamma<\mathrm{SU}(p, 1)$ be a lattice and $\rho: \Gamma \rightarrow \mathrm{PU}(q, 1)$ be a maximal representation. Assume that $p \geq 2$. Then there is an equivariant isometric embedding

which is holomorphic.

$$
F: \mathcal{H}_{\mathbb{C}}^{p} \rightarrow \mathcal{H}_{\mathbb{C}}^{q}
$$

V. Koziarz and J. Maubon gave in [19] a proof of Theorem 2 using harmonic map techniques. We refer to the introduction of their article for an excellent overview of the history and the context of the subject.

The invariant defined above has been introduced in [3] in a much more general situation, in particular for a representation $\rho: \Gamma \rightarrow \operatorname{Iso}(\mathcal{X})^{\circ}$, where $\Gamma<\mathrm{SU}(p, 1)$ is a lattice and $\mathcal{X}$ is now an arbitrary Hermitian symmetric space of noncompact type. In this case $i_{\rho}$ satisfies the inequality

$$
\left|i_{\rho}\right| \leq \operatorname{rank}(\mathcal{X})
$$

and representations for which $i_{\rho}=\operatorname{rank}(\mathcal{X})$ are coined maximal. The case where $p=1$, that is when $\Gamma$ is a surface group, is the object of an ongoing study (see $[12,13,20,16,18,8,6,7,2])$, and in this situation maximal representations lead to new interesting Kleinian groups in higher rank. On the other hand, if $p \geq 2$, we expect maximal representations to come from totally geodesic, possibly holomorphic, embeddings, as it is indeed the case when $\mathcal{X}=\mathcal{H}_{\mathbb{C}}^{q}$.

A different way of looking at $i_{\rho}$ as a foliated Toledo number was suggested to us by F. Labourie, and goes as follows. The space of configurations of points lying on chains can be seen as the space at infinity of the space of configurations

$$
\mathcal{G H}_{\mathbb{C}}^{p}=\left\{(x, Y): Y \text { is a complex geodesic and } x \in Y \subset \mathcal{H}_{\mathbb{C}}^{p}\right\}
$$

of points lying on complex geodesics. This is the total space of a foliation whose leaves are the fibers of the map

$$
\begin{aligned}
\mathrm{pr}_{2}: \mathcal{G H}_{\mathbb{C}}^{p} & \rightarrow \mathcal{G}_{p} \\
(x, Y) & \mapsto Y,
\end{aligned}
$$

where $\mathcal{G}_{p}$ is the set of complex geodesics; the leaves of this foliation are transverse to the fibers of

$$
\begin{aligned}
\operatorname{pr}_{1}: \mathcal{G H}_{\mathbb{C}}^{p} & \rightarrow \mathcal{H}_{\mathbb{C}}^{p} \\
(x, Y) & \mapsto x
\end{aligned}
$$

which, incidentally, are compact. Given now $\Gamma<\mathrm{PU}(p, 1)$ a torsionfree lattice, since $\operatorname{pr}_{1}$ is $\Gamma$-equivariant, we get a foliated space $\operatorname{pr}_{1}: \mathcal{G} M \rightarrow M$ lying above $M=$ $\Gamma \backslash \mathcal{H}_{\mathbb{C}}^{p}$, where $\mathcal{G} M=\Gamma \backslash \mathcal{G} \mathcal{H}_{\mathbb{C}}^{p}$ is foliated by complex geodesics. The restriction to the complex geodesics of the pullback $\operatorname{pr}_{1}^{(2)}\left(\omega_{M}\right)$ of the Kähler form $\omega_{M}$ of $M$, defines a tangential form $\omega_{\mathcal{G} M}$. If then $\rho: \Gamma \rightarrow \mathrm{PU}(q, 1)$ is a homomorphism 
and $\omega_{\rho}^{\prime}$ is a bounded closed representative of the class $\rho^{(2)}\left(\kappa_{q}\right) \in \mathrm{H}_{\mathrm{dR}}^{2}(M)$ (which always exists by [3]), then the tangential form $\Omega_{\rho}^{\prime}$, obtained by restricting $\operatorname{pr}_{1}^{(2)}\left(\omega_{\rho}^{\prime}\right)$ to the leaves of the foliations, differs from $\omega_{\mathcal{G} M}$ by a bounded function, whose integral over $\mathcal{G} M$ gives $i_{\rho}$.

Application to Deformation Rigidity. If $\Gamma$ is a discrete finitely generated group and $L$ is a topological group, the space of homomorphisms $\operatorname{Rep}(\Gamma, L)$ of $\Gamma$ into $L$ is topologized naturally as a closed subset of $L^{S}$, where $S$ is a finite generating set of $\Gamma$. Let $B L$ be the classifying space of continuous principal $L$-bundles, and $c \in \mathrm{H}^{\bullet}(B L, \mathbb{R})$ a characteristic class in degree $\bullet$. It is a standard observation that the map

$$
\begin{aligned}
\operatorname{Rep}(\Gamma, L) & \rightarrow \mathrm{H}^{\bullet}(\Gamma, \mathbb{R}) \\
\rho & \mapsto \rho_{B}^{\bullet}(c),
\end{aligned}
$$

where $\rho_{B}^{\bullet}: \mathrm{H}^{\bullet}(B L, \mathbb{R}) \rightarrow \mathrm{H}^{\bullet}(B \Gamma, \mathbb{R})=\mathrm{H}^{\bullet}(\Gamma, \mathbb{R})$ denotes the pullback, is constant on connected components of $\operatorname{Rep}(\Gamma, L)$.

Assume now that $L$ is simple of Hermitian type and let $K$ be a maximal compact subgroup of $L$. It follows from the Iwasawa decomposition that $B K$ is homotopy equivalent to $B L$, and by Chern-Weil theory $\mathrm{H}^{\bullet}(B K, \mathbb{R})$ is described by the $K$-invariant polynomials on the Lie algebra $\mathfrak{k}$ of $K$, $[11,17]$. Since $L$ is simple Hermitian, the center $Z(\mathfrak{k})$ is one dimensional and the orthogonal projection of $\mathfrak{k}$ on $Z(\mathfrak{k})$ gives rise to an invariant linear form which, via Chern-Weil theory, gives rise to a class in $\mathrm{H}^{2}(B K, \mathbb{R})=\mathrm{H}^{2}(B L, \mathbb{R})$. This class corresponds then via the natural homomorphism $\mathrm{H}^{2}(B L, \mathbb{R}) \rightarrow \mathrm{H}_{\mathrm{c}}^{2}(L, \mathbb{R})$ to the Kähler class $\kappa \mathcal{Y}$, where $\mathcal{Y}$ is the symmetric space associated to $L$, and hence the commutativity of the diagram

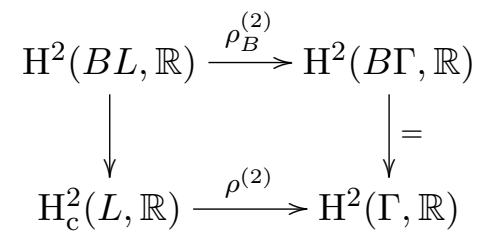

implies that the map

$$
\begin{aligned}
\operatorname{Rep}(\Gamma, L) & \rightarrow \mathrm{H}^{2}(\Gamma, \mathbb{R}) \\
\rho & \mapsto \rho^{(2)}(\kappa \mathcal{Y})
\end{aligned}
$$

is constant on connected components of $\operatorname{Rep}(\Gamma, L)$.

To turn to our immediate application, let us assume that $p \leq q$ and let $\rho_{0}$ : $\mathrm{SU}(p, 1) \rightarrow \mathrm{PU}(q, 1)$ be a standard homomorphism, that is a homomorphism associated to any isometric holomorphic embedding

$$
F: \mathcal{H}_{\mathbb{C}}^{p} \rightarrow \mathcal{H}_{\mathbb{C}}^{q}
$$


Observe that any two such embeddings $\mathcal{H}_{\mathbb{C}}^{p} \rightarrow \mathcal{H}_{\mathbb{C}}^{q}$ are conjugate in $\mathrm{PU}(q, 1)$; moreover, the stabilizer in $\mathrm{PU}(q, 1)$ of the image of $F$ is the almost direct product of the image $\rho_{0}(\mathrm{SU}(p, 1))$ and its centralizer $Z\left(\rho_{0}\right)$ in $\mathrm{PU}(q, 1)$, which is compact.

Corollary 3. Let $\rho_{0}: \mathrm{SU}(p, 1) \rightarrow \mathrm{PU}(q, 1)$ be a standard representation, let $\Gamma<\mathrm{SU}(p, 1)$ be a lattice and assume that $p \geq 2$. Then any representation $\rho: \Gamma \rightarrow \mathrm{PU}(q, 1)$ in the path connected component of $\left.\rho_{0}\right|_{\Gamma}$ in the representation variety $\operatorname{Rep}(\Gamma, \mathrm{PU}(q, 1))$ is, modulo conjugation by $\mathrm{PU}(q, 1)$, of the form $\rho_{0} \times \chi$, where $\chi$ is a homomorphism of $\Gamma$ into the compact group $Z\left(\rho_{0}\right)$.

REMARK 4. We recall that if $\Gamma<\mathrm{SU}(p, 1)$ is cocompact, this was proved by Goldman and Millson in [14]. On the other hand, Gusevskii and Parker found nontrivial deformations of the standard representation $\left.\rho_{0}\right|_{\Gamma}: \Gamma \rightarrow \mathrm{PU}(2,1)$ of a noncocompact lattice: in addition these deformations are quasi-Fuschsian in the sense that they are injective and with discrete image, [15].

\section{The Measurable Cartan Theorem}

Let $V$ be a complex vector space of dimension $\ell+1$ with a Hermitian form $h$ of signature $(\ell, 1)$. The complex hyperbolic $\ell$-space $\mathcal{H}_{\mathbb{C}}^{\ell}$ is the cone of negative lines in $\mathbb{P}(V)$ equipped with the distance

$$
\cosh ^{2} d([v],[w]):=\frac{h(v, w) h(w, v)}{h(v, v) h(w, w)},
$$

which turns it into a simply connected Riemannian manifold with sectional curvature $-4 \leq \kappa \leq-1$ whose connected component of the isometry group is $\mathrm{PU}(\ell, 1)$.

If $0 \leq k \leq \ell$, any $(k+1)$-dimensional nondegenerate indefinite linear subspace $W \subset V$ gives rise to a $k$-plane, that is a totally geodesic holomorphically embedded isometric copy of $\mathcal{H}_{\mathbb{C}}^{k}$. In particular, a 1-plane is a complex geodesic in $\mathcal{H}_{\mathbb{C}}^{\ell}$

The boundary $\partial \mathcal{H}_{\mathbb{C}}^{\ell}$ consists of equivalence classes of asymptotic geodesic rays and can be identified with a $(2 \ell-1)$-dimensional sphere corresponding to the projectivization of the null cone of $h$. Boundaries of $k$-planes are called $k$-chains and boundaries of complex geodesics in $\mathcal{H}_{\mathbb{C}}^{\ell}$ are simply referred to as chains. A chain is completely determined by any two points that belong to it, and hence two distinct chains are either disjoint or meet in exactly one point.

Since the diagonal action on $\operatorname{PU}(\ell, 1)$ on $\left(\partial \mathcal{H}_{\mathbb{C}}^{\ell}\right)^{(3)}$ is not transitive ${ }^{1}$, in the following it will be useful to associate to triples of points in $\left(\partial \mathcal{H}_{\mathbb{C}}^{\ell}\right)^{(3)}$ a full

\footnotetext{
${ }^{1}$ Throughout the paper, given a set $X$ and a positive integer $n \in \mathbb{N}$, we use the notation $X^{(n)}$ to indicate the subset of $X^{n}$ of $n$-ples consisting of distinct points.
} 
invariant which does not have any analog in real hyperbolic spaces except on the hyperbolic plane. Namely, if

$$
\langle\cdot, \cdot, \cdot\rangle:\left(\mathbb{C}^{\ell+1}\right)^{3} \rightarrow \mathbb{C}
$$

is the Hermitian triple product defined by

$$
\left\langle z_{1}, z_{2}, z_{3}\right\rangle=h\left(z_{1}, z_{2}\right) h\left(z_{2}, z_{3}\right) h\left(z_{3}, z_{1}\right),
$$

define Cartan's invariant angulaire

$$
c_{\ell}:\left(\partial \mathcal{H}_{\mathbb{C}}^{\ell}\right)^{(3)} \rightarrow[-1,1]
$$

by

$$
c_{\ell}\left(\xi_{1}, \xi_{2}, \xi_{3}\right):=\frac{2}{\pi} \operatorname{Arg}\left\langle z_{1}, z_{2}, z_{3}\right\rangle,
$$

where the points $z_{i} \in \mathbb{C}^{\ell+1}$ projects onto $\xi_{i} \in \partial \mathcal{H}_{\mathbb{C}}^{\ell}$, and where we choose the convention that $\operatorname{Arg}(z) \in\left[-\frac{\pi}{2}, \frac{\pi}{2}\right],[10]$. Then $c_{\ell}$ extends to a $\mathrm{PU}(\ell, 1)$-invariant alternating cocycle on $\left(\partial \mathcal{H}_{\mathbb{C}}^{\ell}\right)^{3}$; in this section, however, the relevant property of $c_{\ell}$ is that it detects exactly when three points lie of a chain. Namely, $\left|c_{\ell}\left(\xi_{1}, \xi_{2}, \xi_{3}\right)\right|=$ 1 if and only if $\xi_{1}, \xi_{2}, \xi_{3}$ lie on a chain, and $c_{\ell}\left(\xi_{1}, \xi_{2}, \xi_{3}\right)=1$ if and only if the triple $\left(\xi_{1}, \xi_{2}, \xi_{3}\right)$ is positively oriented with respect to the canonical orientation of the chain.

The goal of this section is to prove Theorem 1 . This will be achieved by proving in $\S 2.1$ the following

TheOREM 2.1. Let $p \geq 2$ and let $\varphi: \partial \mathcal{H}_{\mathbb{C}}^{p} \rightarrow \partial \mathcal{H}_{\mathbb{C}}^{q}$ be a measurable map such that:

(i) for almost every chain $C$ and almost every triple $(\xi, \eta, \zeta)$ of distinct points on $C$, the triple $\varphi(\xi), \varphi(\eta), \varphi(\zeta)$ consists also of distinct points which lie on a chain and have the same orientation as $(\xi, \eta, \zeta)$;

(ii) for almost every triple of points $\xi, \eta, \zeta$ not on a chain, $\varphi(\xi), \varphi(\eta), \varphi(\zeta)$ are also not on a chain.

Then there is an isometric holomorphic embedding $F: \mathcal{H}_{\mathbb{C}}^{p} \rightarrow \mathcal{H}_{\mathbb{C}}^{q}$ such that the map

$$
\partial F: \partial \mathcal{H}_{\mathbb{C}}^{p} \rightarrow \partial \mathcal{H}_{\mathbb{C}}^{q}
$$

induced on the boundary coincides with $\varphi$ almost everywhere.

The reduction of Theorem 1 from Theorem 2.1 lies in the following proposition, which will be proven in $\S 2.2$.

Proposition 2.2. Let $\varphi: \partial \mathcal{H}_{\mathbb{C}}^{p} \rightarrow \partial \mathcal{H}_{\mathbb{C}}^{q}$ be a measurable map satisfying (i) but not (ii) of Theorem 2.1. Then there exists a chain $C \subset \partial \mathcal{H}_{\mathbb{C}}^{q}$ such that for almost every $\xi \in \partial \mathcal{H}_{\mathbb{C}}^{p}, \varphi(\xi) \in C$. 
2.1. Proof of Theorem 2.1. The structure of the proof of Theorem 2.1 goes as follows. We first show by induction that the statement of the theorem for a fixed $p$ follows from the analogous statement in one lower dimension, provided $p \geq 3$; this leaves us to show the statement for $p=2$. The next step is to show that if $p=2$ any map $\varphi: \partial \mathcal{H}_{\mathbb{C}}^{2} \rightarrow \partial \mathcal{H}_{\mathbb{C}}^{q}$ satisfying the hypotheses of Theorem 1 essentially takes values in a two-chain; this will be achieved by an appropriate convex hull argument. The last step is hence to show the assertion for $p=q=2$, for which we follow a strategy devised by Goldman for a different proof of E. Cartan's theorem.

2.1.1. Configuration spaces, $I$. In the sequel we will have to deal with various configuration spaces and maps between them. Typically, we will have the situation

$$
p: W \rightarrow V,
$$

where $W, V$ are one of the following configuration spaces below and $p$ is a "projection". In all cases, these configuration spaces are manifolds and the maps $p$ are fibrations. Thus $W, V$ and every fiber of $p$ will be equipped with its canonical Lebesgue measure class, so that the following Fubini-type statement holds: a measurable subset $A \subset W$ is of full measure if and only if for almost every $v \in V$ the set $p^{-1}(v) \cap A$ is of full measure in $p^{-1}(v)$.

We list here for future reference some of the configuration spaces occurring in the part of the proof dealing with the reduction to the case in which $p=q=2$. Let us start by observing that if $\operatorname{Grass}_{k_{1}, k_{2}}(p)$ denotes the Grassmannian of $k_{1}$ planes in $k_{2}$-planes in $\mathcal{H}_{\mathbb{C}}^{p}$, we have

$$
\operatorname{Grass}_{k_{1}, k_{2}}(p) \simeq \mathrm{SU}(p, 1) / \mathrm{S}\left(\mathrm{U}\left(p-k_{2}\right) \times \mathrm{U}\left(k_{2}-k_{1}\right) \times \mathrm{U}\left(k_{1}, 1\right)\right) .
$$

Moreover we will use:

- if $1 \leq k \leq p$, the space $\mathcal{P}_{k, p}$ of $k$-planes in $\mathcal{H}_{\mathbb{C}}^{p}$ and the space $\mathcal{C}_{k, p}$ of $k$-chains in $\partial \mathcal{H}_{\mathbb{C}}^{p}$ are both isomorphic to $\operatorname{Grass}_{k, k}(p)$;

- the space $\left\{(X, x): X \in \mathcal{P}_{p-1, p}, x \in X\right\}$ of points on a $(p-1)$-plane is isomorpic to $\operatorname{Grass}_{0, p-1}(p)$, and the fibers of the projection onto the second factor are the $k$-planes $\mathcal{P}_{k, p}(x)$ through $x \in \mathcal{H}_{\mathbb{C}}^{p}$, where

$$
\mathcal{P}_{k, p}(x) \simeq \mathrm{S}(\mathrm{U}(p) \times \mathrm{U}(1)) / \mathrm{S}(\mathrm{U}(p-k) \times \mathrm{U}(k) \times \mathrm{U}(1)) ;
$$

- the space $\left\{(X, C): X \in \mathcal{P}_{p-1, p}, C \in \mathcal{C}_{k, p}\right.$ and $\left.C \subset \partial X\right\}$ of chains in the boundary of $(p-1)$-planes is isomorphic to $\operatorname{Grass}_{1, p-1}(p)$;

- the space of points in the boundary of a $(p-1)$-plane

$$
\operatorname{Is}_{p-1}(p):=\left\{(X, \xi): X \in \mathcal{P}_{p-1, p}, \xi \in \partial X\right\}
$$

can be identified with

$$
\left\{W \subset X: X \in \mathcal{P}_{k, p}, W \subset X \text { is an isotropic line }\right\},
$$


and as such is isomorphic to

$$
\mathrm{SU}(p, 1) / S\left(U(p-k) \times Q_{k}\right),
$$

where $Q_{k}<\mathrm{SU}(k, 1)$ is a parabolic subgroup stabilizing an isotropic line;

- the space $\left\{\left(X, \xi_{1}, \xi_{2}, \xi_{3}\right): X \in \mathcal{P}_{p-1, p}, \xi_{1}, \xi_{2}, \xi_{3} \in \partial X\right\}$ of triples of points on the boundary of a $(p-1)$-plane is the threefold fibered product $\left(\operatorname{Is}_{p-1}(p)\right)_{f}^{3}$ of the configuration space $\operatorname{Is}_{p-1}(p)$ with respect to the first projection; it turns out to be a manifold, as one can easily see using a standard transversality argument since the projection if submersive;

- the space $\left\{\left(C,\left(\xi_{1}, \xi_{2}\right)\right): C \in \mathcal{C}_{1, p}\right.$ and $\left.\left(\xi_{1}, \xi_{2}\right) \in C^{(2)}\right\}$ is an open set in the twofold fibered product $\left(\operatorname{Is}_{p-1}(p)\right)_{f}^{2}$ which, as in the previous case, is a manifold.

2.1.2. Reduction to the case $p=2$. We now let $p \geq 3$, we assume that the theorem holds for $p-1$ and we will now show that it holds for $p$. Let us start by observing that a simple verification using Fubini's theorem applied to the configuration spaces

$$
\left\{(X, C): X \in \mathcal{P}_{p-1, p}, C \in \mathcal{C}_{1, p}, C \subset \partial X\right\}
$$

and

$$
\left\{\left(X, \xi_{1}, \xi_{2}, \xi_{3}\right): X \in \mathcal{P}_{p-1, p}, \text { and } \xi_{1}, \xi_{2}, \xi_{3} \in \partial X\right\}
$$

shows that, for almost every $X \in \mathcal{P}_{p-1, p}$, the restriction $\left.\varphi\right|_{\partial X}$ of $\varphi$ to $\partial X$ is measurable and satisfies respectively the hypotheses (i) and (ii) of Theorem 2.1.

Applying the induction hypothesis we get for almost every $X \in \mathcal{P}_{p-1, p}$ an isometric holomorphic embedding

$$
F_{X}: X \rightarrow \mathcal{H}_{\mathbb{C}}^{q}
$$

such that $\partial F_{X}=\left.\varphi\right|_{\partial X}$ almost everywhere. To extend $F_{X}$ to a well defined function on $\mathcal{H}_{\mathbb{C}}^{p}$, let us consider the set

$$
\begin{array}{r}
\left\{(x, X): X \in \mathcal{P}_{p-1, p}(x) \text { and there is } F_{X}: X \rightarrow \mathcal{H}_{\mathbb{C}}^{q}\right. \text { as above } \\
\text { with } \left.\partial F_{X}=\left.\varphi\right|_{\partial X} \text { almost everywhere }\right\}
\end{array}
$$

which is thus of full measure in the configuration space

$$
\left\{(x, X): X \in \mathcal{P}_{p-1, p}(x)\right\},
$$

and let us define for almost every $x \in \mathcal{H}_{\mathbb{C}}^{p}$ and almost every $X \in \mathcal{P}_{p-1, p}(x)$ the function

$$
f(x, X):=F_{X}(x) .
$$

Using again Fubini's theorem, one checks that for almost every $X_{1}, X_{2} \in$ $\mathcal{P}_{p-1, p}(x)$,

$$
\left.\partial F_{X_{1}}\right|_{\partial X_{1} \cap \partial X_{2}}=\left.\varphi\right|_{\partial X_{1} \cap \partial X_{2}}=\left.\partial F_{X_{2}}\right|_{\partial X_{1} \cap \partial X_{2}}
$$


For such $X_{1} \neq X_{2}$, since $X_{1} \cap X_{2}$ is nonempty, it is then a $(p-2)$-plane, and since $p-2 \geq 1$, we have that

$$
\partial X_{1} \cap \partial X_{2}=\partial\left(X_{1} \cap X_{2}\right) \neq \emptyset,
$$

which implies, using (2.1), that $f\left(x, X_{1}\right)=f\left(x, X_{2}\right)$. Thus $f(x, X)$ is almost everywhere independent of $X \in \mathcal{P}_{p-1, p}(x)$ and gives rise to a well defined map $f$ : $\mathcal{H}_{\mathbb{C}}^{p} \rightarrow \mathcal{H}_{\mathbb{C}}^{q}$ which by construction preserves the distances of almost every pair of points. It is then easy to see that $f$ coincides almost everywhere with an isometric embedding $\mathcal{H}_{\mathbb{C}}^{p} \rightarrow \mathcal{H}_{\mathbb{C}}^{q}$. This, together with the fact that $\partial f=\varphi$ preserves the orientation on chains, implies that the embedding must be holomorphic.

2.1.3. Reduction to the case $p=q=2$. Recall that any two distinct chains are either disjoint or intersect in a point, and hence every pair of distinct points $(\xi, \eta) \in\left(\partial \mathcal{H}_{\mathbb{C}}^{p}\right)^{(2)}$ determines a unique chain $C(\xi, \eta)$.

Lemma 2.3. Let $\varphi: \partial \mathcal{H}_{\mathbb{C}}^{p} \rightarrow \partial \mathcal{H}_{\mathbb{C}}^{q}$ be a measurable map satisfying the hypothesis (i) of Theorem 2.1 and let $c_{\ell}:\left(\partial \mathcal{H}_{\mathbb{C}}^{\ell}\right)^{3} \rightarrow[-1,1]$ be the Cartan cocycle. Then for almost every $\left(\xi_{1}, \xi_{2}\right) \in\left(\partial \mathcal{H}_{\mathbb{C}}^{p}\right)^{(2)}$, we have that

(1) $\varphi\left(\xi_{1}\right) \neq \varphi\left(\xi_{2}\right)$, and

(2) for almost every $\xi_{3} \in C\left(\xi_{1}, \xi_{2}\right)$, we have

$$
\varphi\left(\xi_{3}\right) \in C\left(\varphi\left(\xi_{1}\right), \varphi\left(\xi_{2}\right)\right)
$$

and

$$
c_{q}\left(\varphi\left(\xi_{1}\right), \varphi\left(\xi_{2}\right), \varphi\left(\xi_{3}\right)\right)=c_{p}\left(\xi_{1}, \xi_{2}, \xi_{3}\right) .
$$

As a consequence we obtain:

Corollary 2.4. Let $\varphi: \partial \mathcal{H}_{\mathbb{C}}^{p} \rightarrow \partial \mathcal{H}_{\mathbb{C}}^{q}$ be a measurable map satisfying the hypothesis (i) of Theorem 2.1. Then there is a measurable map

$$
\Phi: \mathcal{C}_{1, p} \rightarrow \mathcal{C}_{1, q}
$$

such that

$$
\Phi\left(C\left(\xi_{1}, \xi_{2}\right)\right)=C\left(\varphi\left(\xi_{1}\right), \varphi\left(\xi_{2}\right)\right)
$$

for almost every $\left(\xi_{1}, \xi_{2}\right) \in\left(\partial \mathcal{H}_{\mathbb{C}}^{p}\right)^{(2)}$.

Proof of Lemma 2.3. Consider the measure class preserving bijection

$$
\begin{aligned}
\left(\partial \mathcal{H}_{\mathbb{C}}^{p}\right)^{(2)} & \rightarrow\left\{\left(C, \xi_{1}, \xi_{2}\right): C \in \mathcal{C}_{1, p},\left(\xi_{1}, \xi_{2}\right) \in C^{(2)}\right\} \\
\left(\xi_{1}, \xi_{2}\right) & \left.\mapsto C\left(\xi_{1}, \xi_{2}\right), \xi_{1}, \xi_{2}\right) .
\end{aligned}
$$

Then the hypothesis (i) of Theorem 2.1 implies by Fubini that for almost every $C \in \mathcal{C}_{1, p}$, for almost every $\left(\xi_{1}, \xi_{2}\right) \in C^{(2)}$ and for almost every $\xi_{3} \in C$ we have

$$
c_{q}\left(\varphi\left(\xi_{1}\right), \varphi\left(\xi_{2}\right), \varphi\left(\xi_{3}\right)\right)=c_{p}\left(\xi_{1}, \xi_{2}, \xi_{3}\right)
$$


which, using the above bijection, is equivalent to the fact that for almost every $\left(\xi_{1}, \xi_{2}\right) \in\left(\partial \mathcal{H}_{\mathbb{C}}^{p}\right)^{(2)}$ and for almost every $\xi_{3} \in C\left(\xi_{1}, \xi_{2}\right),(2.4)$ holds, which shows that $\varphi\left(\xi_{1}\right) \neq \varphi\left(\xi_{2}\right)$ and that (2) holds.

Proof of Corollary 2.4. It is clear that if $C \in \mathcal{C}_{1, p}$ is such that (2.4) holds for almost every $\left(\xi_{1}, \xi_{2}\right) \in C^{(2)}$ and for almost every $\xi_{3} \in C$, then in particular if $\left(\xi_{1}, \xi_{2}\right) \in C^{(2)}$ and $\left(\eta_{1}, \eta_{2}\right) \in C^{(2)}$ are such that the equalities

$$
\begin{aligned}
c_{q}\left(\varphi\left(\xi_{1}\right), \varphi\left(\xi_{2}\right), \varphi\left(\xi_{3}\right)\right) & =c_{p}\left(\xi_{1}, \xi_{2}, \xi_{3}\right) \\
c_{q}\left(\varphi\left(\eta_{1}\right), \varphi\left(\eta_{2}\right), \varphi\left(\eta_{3}\right)\right) & =c_{p}\left(\eta_{1}, \eta_{2}, \eta_{3}\right)
\end{aligned}
$$

hold for almost every $\xi_{3}, \eta_{3} \in C$, we have that

$$
\begin{aligned}
& \varphi\left(\xi_{1}\right) \neq \varphi\left(\xi_{2}\right), \\
& \varphi\left(\eta_{1}\right) \neq \varphi\left(\eta_{2}\right) \text { and } \\
& C\left(\varphi\left(\xi_{1}\right), \varphi\left(\xi_{2}\right)\right) \cap C\left(\varphi\left(\eta_{1}\right), \varphi\left(\eta_{2}\right)\right) \supset \operatorname{EssIm}\left(\left.\varphi\right|_{C}\right),
\end{aligned}
$$

where $\operatorname{Ess} \operatorname{Im}\left(\left.\varphi\right|_{C}\right)$ denotes the essential image of $\left.\varphi\right|_{C}$. Since $\operatorname{Ess} \operatorname{Im}\left(\left.\varphi\right|_{C}\right)$ cannot be reduced to a point, we have that

$$
C\left(\varphi\left(\xi_{1}\right), \varphi\left(\xi_{2}\right)\right)=C\left(\varphi\left(\eta_{1}\right), \varphi\left(\eta_{2}\right)\right),
$$

which leads to the map

$$
\begin{aligned}
\Phi: \quad \mathcal{C}_{1, p} & \rightarrow \mathcal{C}_{1, q} \\
\left(C\left(\xi_{1}, \xi_{2}\right)\right) & \mapsto C\left(\varphi\left(\xi_{1}\right), \varphi\left(\xi_{2}\right)\right)
\end{aligned}
$$

which is then well defined and satisfies (2.3).

In the sequel we will need a concrete way to choose a probability measure on each chain $C$ which is in the class of the Lebesgue measure. Fix a Riemannian metric on $\partial \mathcal{H}_{\mathbb{C}}^{p}$, for instance the $K$-invariant one, where $K$ is a maximal compact subgroup in $\mathrm{SU}(p, 1)$ : for every $\xi \neq \eta$ in $\partial \mathcal{H}_{\mathbb{C}}^{p}$, if $\mathbb{S}^{1}$ denotes the unit circle in $\mathbb{C}$, let

$$
f_{\xi, \eta}: \mathbb{S}^{1} \rightarrow \partial \mathcal{H}_{\mathbb{C}}^{p}
$$

be the unique parametrization of $C(\xi, \eta)$ such that

$-f_{\xi, \eta}(1)=\xi$

- $f_{\xi, \eta}$ is orientation preserving, and

- $f_{\xi, \eta}$ is a parametrization proportional to the arclength.

If we denote by $\mu_{\xi, \eta} \in \mathcal{M}^{1}\left(\partial \mathcal{H}_{\mathbb{C}}^{p}\right)$ the probability measure supported on $C(\xi, \eta)$ defined by

$$
\mu_{\xi, \eta}(F):=\frac{1}{2 \pi} \int_{0}^{2 \pi} F\left(f_{\xi, \eta}\left(e^{i \theta}\right)\right) d \theta
$$


then the map

$$
\begin{aligned}
\left(\partial \mathcal{H}_{\mathbb{C}}^{p}\right)^{(2)} & \rightarrow \mathcal{M}^{1}\left(\partial \mathcal{H}_{\mathbb{C}}^{p}\right) \\
\left(\xi_{1}, \xi_{2}\right) & \mapsto \mu_{\left(\xi_{1}, \xi_{2}\right)},
\end{aligned}
$$

is clearly continuous for the weak-* topology on $\mathcal{M}^{1}\left(\partial \mathcal{H}_{\mathbb{C}}^{p}\right)$. Then we have:

Lemma 2.5. Let $\lambda$ be the $K$-invariant probability measure on $\partial \mathcal{H}_{\mathbb{C}}^{p}$. Then for every $\xi_{1} \in \partial \mathcal{H}_{\mathbb{C}}^{p}$, the probability measure on $\partial \mathcal{H}_{\mathbb{C}}^{p} \times \partial \mathcal{H}_{\mathbb{C}}^{p}$ defined by

$$
f \mapsto \int_{\partial \mathcal{H}_{\mathbb{C}}^{p}} \int_{\partial \mathcal{H}_{\mathbb{C}}^{p}}\left(\mu_{\left(\xi_{1}, \xi_{2}\right)} \otimes \mu_{\left(\xi_{1}, \xi_{3}\right)}\right)(f) d \lambda\left(\xi_{2}\right) d \lambda\left(\xi_{3}\right)
$$

for any continuous function $f$ on $\partial \mathcal{H}_{\mathbb{C}}^{p} \times \partial \mathcal{H}_{\mathbb{C}}^{p}$, is equivalent to $\lambda \otimes \lambda$.

Proof. The measure defined above is the product with itself of the measure on $\partial \mathcal{H}_{\mathbb{C}}^{p}$ given by

$$
f \mapsto \int_{\partial \mathcal{H}_{\mathbb{C}}^{p}} \frac{1}{2 \pi} \int_{0}^{2 \pi} f\left(f_{\xi_{1}, \eta}\left(e^{i \theta}\right)\right) d \theta d \lambda(\eta),
$$

which is equivalent to $\lambda$ since the chains through $\xi_{1}$ give a smooth foliation of $\partial \mathcal{H}_{\mathbb{C}}^{p} \backslash\left\{\xi_{1}\right\}$.

Lemma 2.6. Let $\xi_{1} \in \partial \mathcal{H}_{\mathbb{C}}^{p}$. For almost every $\left(\xi_{2}, \xi_{3}\right) \in\left(\partial \mathcal{H}_{\mathbb{C}}^{p}\right)^{(2)}$, for almost every $(a, b) \in C\left(\xi_{1}, \xi_{2}\right) \times C\left(\xi_{1}, \xi_{3}\right)$ and for almost every $c \in C(a, b)$, we have that:

$$
\begin{aligned}
& \varphi(a) \in C\left(\varphi\left(\xi_{1}\right), \varphi\left(\xi_{2}\right)\right) ; \\
& \varphi(b) \in C\left(\varphi\left(\xi_{1}\right), \varphi\left(\xi_{3}\right)\right) ; \\
& \varphi(c) \in C(\varphi(a), \varphi(b)) .
\end{aligned}
$$

Proof. According to Lemma 2.3(2), the set

$$
\begin{array}{r}
E:=\left\{(a, b) \in\left(\partial \mathcal{H}_{\mathbb{C}}\right)^{2}: \text { for almost every } c \in C(a, b),\right. \\
\text { we have } \varphi(c) \in C(\varphi(a), \varphi(b))\}
\end{array}
$$

is of full $\lambda \otimes \lambda$-measure. Thus, by Lemma 2.5 , we have

$$
1=\int_{\partial \mathcal{H}_{\mathbb{C}}^{p}} \int_{\partial \mathcal{H}_{\mathbb{C}}^{p}}\left(\mu_{\left(\xi_{1}, \xi_{2}\right)} \otimes \mu_{\left(\xi_{1}, \xi_{3}\right)}\right)(E) d \lambda\left(\xi_{2}\right) d \lambda\left(\xi_{3}\right)
$$

which, taking into account that all measures involved are probability measures, is equivalent to the conclusion of Lemma 2.6.

Corollary 2.7. Let $\varphi: \partial \mathcal{H}_{\mathbb{C}}^{2} \rightarrow \partial \mathcal{H}_{\mathbb{C}}^{q}$ be a measurable map satisfying the hypothesis (i) of Theorem 2.1. Then the essential image of $\varphi$ is contained in a 2-chain. 
Proof. Fix $\left(\xi_{1}, \xi_{2}, \xi_{3}\right)$ not on a chain, for which Lemma 2.6 holds. Let $E \subset \partial \mathcal{H}_{\mathbb{C}}^{2}$ be the set of $c \in \partial \mathcal{H}_{\mathbb{C}}^{2}$ such that there are $(a, b) \in C\left(\xi_{1}, \xi_{2}\right) \times C\left(\xi_{1}, \xi_{3}\right)$ with $c \in C(a, b)$ and Lemma 2.6 holds for $a, b, c$. Then $E \subset \partial \mathcal{H}_{\mathbb{C}}^{2}$ is of full measure. Moreover,

$\varphi(c)$ is in the $\mathbb{C}$-linear span of $\varphi(a)$ and $\varphi(b)$,

$\varphi(a)$ is in the $\mathbb{C}$-linear span of $\varphi\left(\xi_{1}\right)$ and $\varphi\left(\xi_{2}\right)$, and

$\varphi(b)$ is in the $\mathbb{C}$-linear span of $\varphi\left(\xi_{1}\right)$ and $\varphi\left(\xi_{3}\right)$,

so that for all $c \in E, \varphi(c)$ is in the 2-chain determined by the 3-dimensional space $\varphi\left(\xi_{1}\right) \oplus \varphi\left(\xi_{2}\right) \oplus \varphi\left(\xi_{3}\right)$.

2.1.4. Configuration spaces, II. In the last part of the proof we will need some more configuration spaces which, as manifolds, will come equipped as before with a natural Lebesgue measure class. We will use:

- the space $\Pi$ of circles of positive radius in $\mathbb{C}$, isomorphic to $\mathrm{Aff}(\mathbb{C}) / \mathbb{C}_{1}^{\star} \simeq$ $\mathbb{C}^{*} \ltimes \mathbb{C} / \mathbb{C}_{1}^{\star}$;

- the space of points on a circle $\Delta=\{(S, z) ; z \in S, S$ a circle in $\mathbb{C}\}$, isomorphic to $\operatorname{Aff}(\mathbb{C})$;

- the space of triples of distincts points on a circle, which is an open set in the fibered product

$$
(\Delta)_{f}^{3}:=\left\{\left(S, z_{1}, z_{2}, z_{3}\right): C \text { is a circle in } \mathbb{C},\left(z_{1}, z_{2}, z_{3}\right) \in C^{3}\right\}
$$

with respect to the projection on the second component.

Since circles through a point correspond to affine lines via an inversion, we will also use the following configurations:

- the space $\mathcal{D}$ of lines in $\mathbb{C}$, which is isomorphic to $\operatorname{Aff}(\mathbb{C}) / \mathbb{R}^{*} \ltimes \mathbb{R}$;

- the space $\mathcal{D}(w)$ of lines in $\mathbb{C}$ through the point $w \in \mathbb{C}$;

- the space $\mathcal{K}_{0}:=\{(d, L): L \in d, d \in \mathcal{D}\}$ of points on a line, isomorphic to $\operatorname{Aff}(\mathbb{C})$;

- the space $\mathcal{K}:=\left\{(d, A, B, C): d \in \mathcal{D}\right.$, and $\left.(A, B, C) \in d^{(3)}\right\}$ is an open set in the manifold

$$
\left\{(d, A, B, C): d \in \mathcal{D} \text {, and }(A, B, C) \in d^{3}\right\} \simeq\left(\mathcal{K}_{0}\right)_{f}^{3},
$$

where the fibered porduct is with respect to the projection on the first component $p_{1}$;

- the space

$$
\begin{array}{r}
\mathcal{K}_{1}:=\left\{\left(d^{\prime}, d, A, B, C, M\right):(d, A, B, C) \in \mathcal{K},\left(d^{\prime}, A\right) \in \mathcal{K}_{0},\right. \\
\left.d^{\prime} \neq d, M \notin d^{\prime} \cup d\right\},
\end{array}
$$


which is an open set in

$$
\begin{aligned}
& \left(\mathcal{K} \times \mathcal{K}_{0}\right)_{f} \times \mathbb{C}:= \\
& \left\{\left(d^{\prime}, A, B, C, d, M\right):(d, A, B, C) \in \mathcal{K},\left(d^{\prime}, A\right) \in \mathcal{K}_{0},\right\}
\end{aligned}
$$

where

$$
\begin{array}{r}
\left(\mathcal{K} \times \mathcal{K}_{0}\right)_{f}:=\left\{\left((d, A, B, C),\left(d^{\prime}, L\right)\right) \in \mathcal{K} \times \mathcal{K}_{0}:\right. \\
\left.p_{2}(d, A, B, C)=p_{2}\left(d^{\prime}, L\right)\right\} .
\end{array}
$$

\subsubsection{The Case $p=q=2$.}

Proof of Theorem 2.1. Let us denote by $\mathcal{C}_{1,2}(\xi)$ the set of chains through the point $\xi \in \partial \mathcal{H}_{\mathbb{C}}^{2}$ and let now $\varphi: \partial \mathcal{H}_{\mathbb{C}}^{2} \rightarrow \partial \mathcal{H}_{\mathbb{C}}^{2}$ be a measurable map satisfying the hypotheses of Theorem 2.1 for $p=q=2$. Let $\Phi: \mathcal{C}_{1,2} \rightarrow \mathcal{C}_{1,2}$ be the map induced almost everywhere on the set of chains defined in (2.2), and let $E \subset \partial \mathcal{H}_{\mathbb{C}}^{2}$ be the subset of full measure such that for every $\xi \in E$ and almost every $C \in \mathcal{C}_{1,2}(\xi)$, also $\Phi(C) \in \mathcal{C}_{1,2}(\varphi(\xi))$. Fix $\xi \in E$; composing with an element from $\mathrm{SU}(2,1)$, we may assume that $\varphi(\xi)=\xi$. The idea of the proof consists in considering a quotient map

$$
\pi_{\xi}: \partial \mathcal{H}_{\mathbb{C}}^{2} \backslash\{\xi\} \rightarrow \mathbb{C}
$$

whose fibers are the chains through $\xi$ (with $\xi$ removed). Then, if $g_{\xi}: \mathbb{C} \rightarrow \mathbb{C}$ denotes the induced measurable map such that the diagram

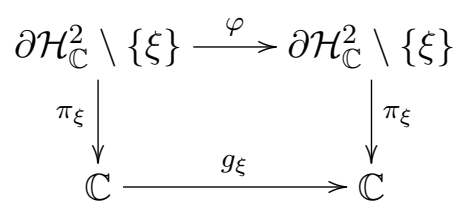

commutes, information about the behavior of the map $\varphi$ in the direction transverse to the fiber of $\pi_{\xi}$ will allow to conclude that $g_{\xi}$ is an affine map of $\mathbb{C}$. From this, adjusting appropriately the map $\varphi$ via elements of $\mathrm{SU}(2,1)$, we will conclude that $\varphi=I d$ almost everywhere, and hence obtain the conclusion of the theorem.

To this purpose, let $P$ be the stabilizer in $\mathrm{SU}(2,1)$ of $\xi$, and let $N$ be its unipotent radical. Then $\partial \mathcal{H}_{\mathbb{C}}^{2} \backslash\{\xi\}$ is a principal homogeneous $N$-space, and the orbits of the center $Z(N)$ are the chains through $\xi$ (with the point $\xi$ removed). Since $N / Z(N)$ can be identified with $\mathbb{C}$, the quotient map $N \rightarrow N / Z(N)$ induces a map

$$
\pi_{\xi}: \partial \mathcal{H}_{\mathbb{C}}^{2} \backslash\{\xi\} \rightarrow \mathbb{C}
$$

whose fibers are the chains through $\xi$ and which enjoys the following properties:

(P1) It is equivariant with respect to the homomorphism

$$
\omega_{\xi}: P \rightarrow \operatorname{Aff}(\mathbb{C})
$$


induced from the identification

$$
P / Z(N) \stackrel{\cong}{\longrightarrow} \operatorname{Aff}(\mathbb{C}) .
$$

(P2) for every chain $C \subset \partial \mathcal{H}_{\mathbb{C}}^{2} \backslash\{\xi\},\left.\pi_{\xi}\right|_{C}$ is injective with image a circle in $\mathbb{C}$;

(P3) for every circle $S \subset \mathbb{C}$ and any $s \in \partial \mathcal{H}_{\mathbb{C}}^{2} \backslash\{\xi\}$ with $\pi_{\xi}(s) \in S$, there is a (unique) chain $C \subset \partial \mathcal{H}_{\mathbb{C}}^{2} \backslash\{\xi\}$ through $s$ such that $\pi_{\xi}(C)=S$.

Moreover, $g_{\xi}$ induces a measurable map $G_{\xi}: \Pi \rightarrow \Pi$ from the set $\Pi$ of circles in $\mathbb{C}$ into itself. To continue, we are going to need the following result, whose proof we postpone.

Proposition 2.8. Let $g: \mathbb{C} \rightarrow \mathbb{C}$ be a measurable map and let us assume that there exists a measurable map $G: \Pi \rightarrow \Pi$ such that for almost every circle $S \subset \mathbb{C}$, there is a circle $G(S) \subset \mathbb{C}$ such that:

(i) for almost every $z \in S, g(z) \in G(S)$;

(ii) for almost every $z_{1}, z_{2}, z_{3} \in S$ distinct, $g\left(z_{1}\right), g\left(z_{2}\right), g\left(z_{3}\right) \in G(S)$ are distinct and in the same cyclic order;

(iii) for almost every $z \in \mathbb{C}$ the set

$$
\left\{\left(S_{1}, S_{2}\right): z \in S_{i}, i=1,2, G\left(S_{1}\right)=G\left(S_{2}\right)\right\}
$$

is of measure zero.

Then $g$ coincides almost everywhere with an affine map $z \mapsto \lambda z+c$, where $\lambda \in \mathbb{C}^{\times}$ and $c \in \mathbb{C}$.

Continuation of the proof of Theorem 2.1. Since two chains which intersect in two points must coincide, and because of properties (P1), (P2) and (P3), then $g_{\xi}$ satisfies the hypotheses of the above proposition and hence $g_{\xi} \in \operatorname{Aff}(\mathbb{C})$.

Thus composing $\varphi$ with an element $h^{-1} \in P$ such that $\omega_{\xi}(h)=g_{\xi}$ almost everywhere, we may assume that $g_{\xi}=I d$ almost everywhere, that is, for almost every $C \in \mathcal{C}_{1,2}(\xi)$ and almost every $\zeta \in C, \varphi(\zeta) \in C$. Now pick such a $C$ and $\eta \in C \cap E$, where $E$ has been defined at the beginning of the proof. Composing with an element from $Z(N)$, we may assume that $\varphi(\eta)=\eta$. But then the map $g_{\eta}: \mathbb{C} \rightarrow \mathbb{C}$ fixes $\pi_{\eta}(\xi)$, and since $\varphi$ leaves invariant all chains through $\xi$, then $g_{\eta}$ leaves invariant all circles through $\pi_{\eta}(\xi)$; this, together with the fact that by Proposition 2.8 again $g_{\eta}$ coincides almost everywhere with an affine map, implies that $g_{\eta}=I d_{\mathbb{C}}$. But $\pi_{\eta}$ is injective when restricted to every chain $C \in \mathcal{C}_{1,2}(\xi)$ different from $C(\xi, \eta)$ and, since $g_{\eta}=I d_{\mathbb{C}}$, it follows that $\left.\varphi\right|_{C}=\left.I d\right|_{C}$. Since the chains through $\xi$ but not through $\eta$ foliate a set of $\partial \mathcal{H}_{\mathbb{C}}^{2}$ of full measure, we conclude that $\varphi$ coincides almost everywhere with the identity.

Proof of Proposition 2.8. By Fubini's theorem, the set $E$ of $z \in \mathbb{C}$ such that (i), (ii) and (iii) hold for almost every circle through $z$ is of full measure in $\mathbb{C}$; 
fixing $z \in E$ and composing with an affine map, we may assume that $g(z)=z$. Conjugating $g$ with an inversion $i: \widehat{\mathbb{C}} \rightarrow \widehat{\mathbb{C}}$ with respect to a circle with center $z$, we get a map $f: \mathbb{C} \rightarrow \mathbb{C}$ which induces a map $F: \mathcal{D} \rightarrow \mathcal{D}$ on the set $\mathcal{D}$ of affine $\mathbb{R}$-lines in $\mathbb{C}$, satisfying the following properties:

(i)' for almost every $d \in \mathcal{D}$ and almost every $P \in d$, we have that $f(P) \in F(d)$;

(ii)' for almost every $d \in \mathcal{D}$ and almost every $A, B, C \in d$ distinct, the points $f(A), f(B), f(C)$ are distinct and lie on $F(d)$;

(iii)' for almost every point $w \in \mathbb{C}$, the set

$$
\left\{\left(d_{1}, d_{2}\right) \in \mathcal{D}(w)^{2}: F\left(d_{1}\right)=F\left(d_{2}\right)\right\}
$$

is of measure zero in $(\mathcal{D}(w))^{2}$.

Notice that in (ii)' one could have stated also a condition corresponding to the preservation of the cyclic ordering. However this is never used for the maps $f$ and $F$ themselves and comes into play only at the very end of the proof when we return to the map $g$, so that we chose to ignore it.

The main point of the proof is the following claim, which we assume for the moment and prove later:

Claim. If $f$ and $F$ are as above, then $f$ preserves almost everywhere the property for four-tuples to be in anharmonic position: namely, if

$$
\left[z_{1}, z_{2}, z_{3}, z_{4}\right]:=\frac{\left(z_{1}-z_{3}\right)\left(z_{2}-z_{4}\right)}{\left(z_{2}-z_{3}\right)\left(z_{1}-z_{4}\right)}
$$

denotes the crossratio, and if, as in $\S 2.1 .4$,

$$
\mathcal{K}:=\{(d, A, B, C): d \in \mathcal{D} \text {, and } A, B, C \text { are distinct points on } d\},
$$

then for almost every $(d, A, B, C) \in \mathcal{K}$, if $D \in d$ and $D^{\prime} \in F(d)$ are points such that $[A, B, C, D]=-1$ and $\left[f(A), f(B), f(C), D^{\prime}\right]=-1$, then

$$
f(D)=D^{\prime}
$$

Observe here that $\mathcal{K}$ is a manifold and, as such, is endowed with the Lebesgue measure class. Assuming this claim, let us define

$$
\mathcal{V}_{-1}:=\left\{\left(z_{1}, z_{2}, z_{3}, z_{4}\right) \in \mathbb{C}^{4}:\left[z_{1}, z_{2}, z_{3}, z_{4}\right]=-1\right\} .
$$

Then $\mathcal{V}_{-1}$ is a three-dimensional complex manifold isomorphic to $\mathbb{C}^{(3)}$ via any of the four projections

$$
\operatorname{pr}_{i}: \mathcal{V}_{-1} \rightarrow \mathbb{C}^{(3)}
$$

consisting of dropping the $i$-th coordinate, for $i=1, \ldots, 4$. The original map $g$ has the property that

$$
\text { for a.e. }\left(z_{1}, z_{2}, z_{3}, z_{4}\right) \in \mathcal{V}_{-1}
$$

we have that $\left(g\left(z_{1}\right), g\left(z_{2}\right), g\left(z_{3}\right), g\left(z_{4}\right)\right) \in \mathcal{V}_{-1}$, 
where the almost everywhere statement is with respect to the natural Lebesgue measure on $\mathcal{V}_{-1}$.

By Fubini's theorem, one can fix $z_{4} \in \mathbb{C}$ such that if

$$
\mathcal{V}_{-1}^{\left(z_{4}\right)}:=\left\{\left(z_{1}, z_{2}, z_{3}\right) \in \mathbb{C}:\left[z_{1}, z_{2}, z_{3}, z_{4}\right]=-1\right\},
$$

then for almost every $\left(z_{1}, z_{2}, z_{3}\right) \in \mathcal{V}_{-1}^{\left(z_{4}\right)}$ the quadruple $\left(g\left(z_{1}\right), g\left(z_{2}\right), g\left(z_{3}\right), g\left(z_{4}\right)\right)$ is in $\mathcal{V}_{-1}$. Consider the composition

$$
\tilde{g}:=i \circ g \circ j,
$$

where $i$ and $j$ are inversions with $j(\infty)=z_{4}$ and $i\left(g\left(z_{4}\right)\right)=\infty$. Then (2.6) holds with $g$ replaced by $\tilde{g}$ and $z_{4}$ replaced by $\infty$, so that, using the definition of crossratio, one has that for almost every $\left(z_{1}, z_{2}\right) \in \mathbb{C}^{2}$

$$
2 \tilde{g}\left(\frac{z_{1}+z_{2}}{2}\right)=\tilde{g}\left(z_{1}\right)+\tilde{g}\left(z_{2}\right) \text {. }
$$

We claim that this implies that $\tilde{g}$ is an $\mathbb{R}$-affine transformation of $\mathbb{C}$. To this purpose, define $h: \mathbb{C} \times \mathbb{C} \rightarrow \mathbb{C}$ by

$$
h\left(z_{1}, z_{2}\right):=\tilde{g}\left(z_{1}\right)-\tilde{g}\left(z_{2}\right) .
$$

Using equation (2.7), one can easily see that for almost every $\left(z_{1}, z_{2}\right) \in \mathbb{C}^{2}$, the map

$$
\begin{aligned}
& \mathbb{C} \longrightarrow \mathbb{C} \\
& u \mapsto h\left(u+z_{1}, u+z_{2}\right)
\end{aligned}
$$

is almost everywhere constant, which implies that there exists a measurable map $\tilde{h}: \mathbb{C} \rightarrow \mathbb{C}$ such that

$$
\tilde{h}\left(z_{1}-z_{2}\right)=\tilde{g}\left(z_{1}\right)-\tilde{g}\left(z_{2}\right)
$$

for almost every $\left(z_{1}, z_{2}\right) \in \mathbb{C}^{2}$.

Applying this equation (2.8) to the pairs $\left(z_{1}, z_{2}\right),\left(z_{2}, z_{3}\right)$ and $\left(z_{1}, z_{3}\right)$, one sees that $\tilde{h}$ is a measurable homomorphism of the additive group $(\mathbb{C},+)$, and hence coincides almost everywhere with an $\mathbb{R}$-linear map, which in turns, in view of (2.8), implies that $\tilde{g}$ is $\mathbb{R}$-affine. But since $\tilde{g}$ sends circles to circles, it is either $\mathbb{C}$-affine or $\overline{\mathbb{C}}$-affine, so that $g$ is either a homography or an antihomography. But then, using that $g$ has to preserve cyclic order on circles, one concludes that $g$ is $\mathbb{C}$-affine.

Proof of Claim. If $f: \mathbb{C} \rightarrow \mathbb{C}$ were an everywhere defined map which satisfies (i)', (ii)', and (iii)' pointwise, then the statement of the claim would be nothing but an application of la méthode du quadrilatère complet which we recall here [9].

Let $A, B, C$ be three distinct points on a line $d$ and, given another line $d^{\prime} \neq d$, and a point $M \notin d \cup d^{\prime}$, we construct a fourth point $D \in d$ such that $[A, B, C, D]=$ -1 . To this purpose, let $P:=d_{C M} \cap d^{\prime}$, where $d_{C M}$ is the line determined by the 


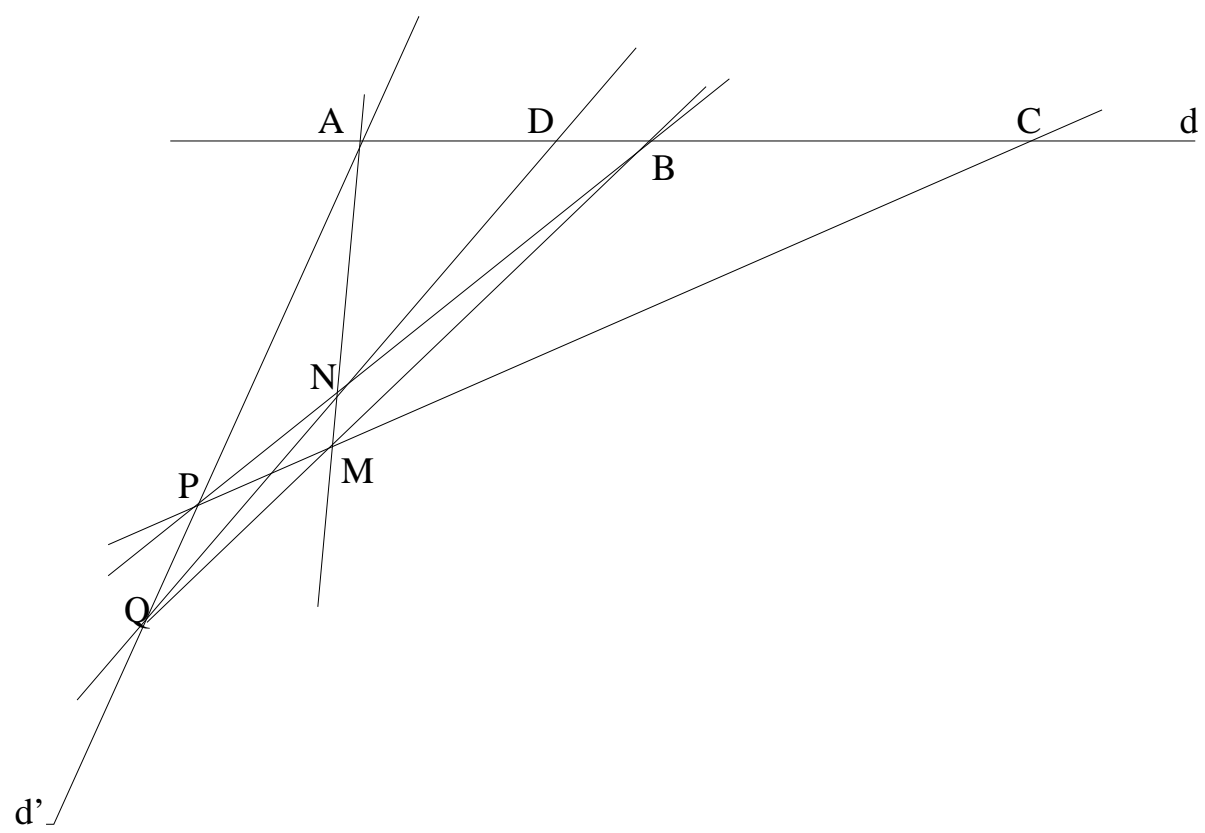

Figure 1. La méthode du quadrilatère complet.

points $C$ and $M$, and let $Q:=d_{B M} \cap d^{\prime}$. If $N:=d_{P B} \cap d_{A M}$, then $D:=d_{Q N} \cap d$ is the unique point such that $[A, B, C, D]=-1$. Repeated applications of the properties (i)', (ii)', and (iii)' imply that $[f(A), f(B), f(C), f(D)]=-1$.

For a measurable function $f: \mathbb{C} \rightarrow \mathbb{C}$ for which all of the above statements are true almost everywhere, the first thing to verify is that the statement of the claim makes sense. This is however true since properties (i)' and (ii)' imply that for almost every $(d, A, B, C) \in \mathcal{K}$, one has that

$$
(F(d), f(A), f(B), f(C)) \in \mathcal{K}
$$

as well.

Let, as in $\S 2.1 .4$,

$$
\mathcal{K}_{0}:=\{(d, A) ; d \in \mathcal{D}, A \in d\}
$$

and let

$$
\begin{array}{r}
\mathcal{K}_{1}:=\left\{\left(d^{\prime}, d, A, B, C, M\right):(d, A, B, C) \in \mathcal{K},\left(d^{\prime}, A\right) \in \mathcal{K}_{0}\right. \\
\text { and } \left.d^{\prime} \neq d, M \notin d^{\prime} \cup d\right\} .
\end{array}
$$

Like $\mathcal{K}$, the spaces $\mathcal{K}_{0}$ and $\mathcal{K}_{1}$ are manifolds and are hence endowed with their Lebesgue measure class. First we observe that properties (ii)' and (iii)' together with a repeated use of Fubini's theorem imply that there is a subset $E_{1} \subset \mathcal{K}_{1}$ of 
full measure such that for all $\left(d^{\prime}, d, A, B, C, M\right) \in E_{1}$, then

$$
\left(F\left(d^{\prime}\right), F(d), f(A), f(B), f(C), f(M)\right) \in \mathcal{K}_{1} .
$$

Now consider the following maps

$$
m_{i}: \mathcal{K}_{1} \rightarrow \mathcal{K}
$$

for $i=1, \ldots, 7$, given by

$$
\begin{aligned}
& m_{1}:\left(d^{\prime}, d, A, B, C, M\right) \mapsto\left(d_{C M}, C, M, P\right) \\
& m_{2}:\left(d^{\prime}, d, A, B, C, M\right) \mapsto\left(d_{B M}, B, M, N\right) \\
& m_{3}:\left(d^{\prime}, d, A, B, C, M\right) \mapsto\left(d_{A M}, A, Q, M\right) \\
& m_{4}:\left(d^{\prime}, d, A, B, C, M\right) \mapsto\left(d_{P B}, P, Q, B\right) \\
& m_{5}:\left(d^{\prime}, d, A, B, C, M\right) \mapsto\left(d^{\prime}, A, P, N\right) \\
& m_{6}:\left(d^{\prime}, d, A, B, C, M\right) \mapsto\left(d_{N Q}, N, Q, D\right) \\
& m_{7}:\left(d^{\prime}, d, A, B, C, M\right) \mapsto(d, A, B, D),
\end{aligned}
$$

Let $T \subset \mathcal{K}$ be the subset consisting of quadruples $(d, A, B, C)$ such that the triple $(f(A), f(B), f(C))$ consists of pairwise distinct points and belongs to $F(d)$, that is $(F(d), f(A), f(B), f(C)) \in \mathcal{K}$. Then, because of (2.9), the set $T$ is of full measure and it is easy to verify that then $m_{i}^{-1}(T) \subset \mathcal{K}_{1}$ is of full measure for $i=1, \ldots, 7$. Thus the same is true for

$$
E_{1}^{\prime}:=\bigcap_{i=1}^{7} m_{i}^{-1}(T) \cap E_{1} .
$$

But then the previous pointwise argument shows that for all confgurations of points and lines $\left(d^{\prime}, d, A, B, C, M\right) \in E_{1}^{\prime}$,

$$
\begin{array}{clrl}
\text { if } D \in d & \text { is such that } & {[A, B, C, D]} & =-1 \\
\text { and } D^{\prime} \in F(d) & \text { is such that }\left[f(A), f(B), f(C), D^{\prime}\right] & =-1
\end{array}
$$

then $f(D)=D^{\prime}$.

2.2. Proof of Proposition 2.2. We start with the following

Lemma 2.9. Let $B \subset \partial \mathcal{H}_{\mathbb{C}}^{p}$ be a measurable set of positive measure such that for almost every $(\xi, \eta) \in B^{(2)}$, the set $C(\xi, \eta) \cap B$ is of full measure in the chain $C(\xi, \eta)$. Then $B$ is a set of full measure in $\partial \mathcal{H}_{\mathbb{C}}^{p}$.

Proof. We may pick a density point $\xi \in B$ such that for almost every $\eta \in B$, the set $C(\xi, \eta) \cap B$ has full measure in $C(\xi, \eta)$. Now let

$$
\mathcal{C}_{B}:=\{C \in \mathcal{C}(\xi): C \cap B \text { has positive measure in } C\} .
$$


Then by Fubini's theorem we have that for almost every $C \in \mathcal{C}_{B}$ and almost every $\eta \in C \cap B$, the set $C(\xi, \eta) \cap B$ is of full measure in $C(\xi, \eta)$ and hence, for all $C \in \mathcal{C}_{B}$, we have that

$$
C \cap B \text { is of full measure in } C \text {. }
$$

On the other hand, using that $\xi$ is a density point, it is easy to see that $\mathcal{C}_{B}$ has full measure in $\mathcal{C}(\xi)$ and hence, again by (2.10) and Fubini's theorem, $B$ has full measure in $\partial \mathcal{H}_{\mathbb{C}}^{p}$.

Proof of Proposition 2.2. If (ii) fails, then the set

$$
\{(\xi, \eta, \zeta): \varphi(\xi), \varphi(\eta), \varphi(\zeta) \text { are on a chain }\}
$$

is of positive measure in $\partial \mathcal{H}_{\mathbb{C}}^{p}$. Observing by (i) that for almost every $(\xi, \eta) \in$ $\left(\partial \mathcal{H}_{\mathbb{C}}^{p}\right)^{2}$ we have that $\varphi(\xi) \neq \varphi(\eta)$, we deduce by Fubini's theorem that there exists $(\xi, \eta)$ such that

(1) $\varphi(\xi) \neq \varphi(\eta)$, and

(2) the set of $\left\{\zeta \in \partial \mathcal{H}_{\mathbb{C}}^{p}\right.$ such that $\varphi(\xi), \varphi(\eta), \varphi(\zeta)$ are on a chain $\}$ is of positive measure.

Denoting by $C$ the chain through $\varphi(\xi)$ and $\varphi(\eta)$, we conclude that $B:=\varphi^{-1}(C)$ is of positive measure and, in view of the hypothesis (i), satisfies the assumptions of Lemma 2.9, which shows that $\varphi^{-1}(C)$ is of full measure.

\section{Proof of Theorem 2}

Let $\Gamma<\mathrm{SU}(p, 1)$ be a lattice, $\rho: \Gamma \rightarrow \mathrm{PU}(q, 1)$ a homomorphism with nonelementary image and let $\varphi: \partial \mathcal{H}_{\mathbb{C}}^{p} \rightarrow \partial \mathcal{H}_{\mathbb{C}}^{q}$ be the $\Gamma$-equivariant measurable boundary map. For almost every chain $C \in \mathcal{C}_{1, p}$ let us denote by $\varphi_{C}$ the restriction of $\varphi$ to $C$. Denoting by $\mu$ the $\mathrm{SU}(p, 1)$-invariant probability measure on $\Gamma \backslash \mathrm{SU}(p, 1)$, we established in [4] the following formula which gives a measure of how much the boundary map $\varphi$ distorts a typical chain:

Theorem 3.1 ([4, Theorem 5.6 and Corollary 5.7]). Let $\Gamma<\mathrm{SU}(p, 1)$ be a lattice, $\rho: \Gamma \rightarrow \mathrm{PU}(q, 1)$ a homomorphism with nonelementary image and $\varphi: \partial \mathcal{H}_{\mathbb{C}}^{p} \rightarrow$ $\partial \mathcal{H}_{\mathbb{C}}^{q}$ be the associated $\Gamma$-equivariant measurable boundary map. For almost every chain $C \in \mathcal{C}_{1, p}$ and almost every triple $(\xi, \eta, \zeta) \in C^{3}$, we have

$$
\int_{\Gamma \backslash \mathrm{SU}(p, 1)} c_{q}\left(\varphi_{C}(g \xi), \varphi_{C}(g \eta), \varphi_{C}(g \zeta)\right) d \mu(\dot{g})=i_{\rho} c_{p}(\xi, \eta, \zeta),
$$

where $i_{\rho}$ is defined in (1.1), and $c_{q}, c_{p}$ are the Cartan cocycles. 
If $i_{\rho}=1$ then for almost every $C \in \mathcal{C}_{1, p}$ and almost every $\left(\xi_{1}, \xi_{2}, \xi_{3}\right) \in C^{3}$

$$
c_{q}\left(\varphi_{C}\left(\xi_{1}\right), \varphi_{C}\left(\xi_{2}\right), \varphi_{C}\left(\xi_{3}\right)\right)=c_{p}\left(\xi_{1}, \xi_{2}, \xi_{3}\right) .
$$

Proof of Theorem 2. We start by observing that since $i_{\rho}=1$, then $\rho(\Gamma)<$ $\operatorname{PU}(q, 1)$ is nonelementary. In fact, if $\rho(\Gamma)$ were elementary, it would be contained in a closed amenable subgroup of $\mathrm{PU}(1,1)$. Since the class $\kappa_{q}$ is bounded, its restriction to any closed amenable subgroup vanishes; thus elementarity of $\rho(\Gamma)$ would imply that $\rho^{(2)}\left(\kappa_{q}\right)=0$, and hence $i_{\rho}=0$.

Let now $\varphi: \partial \mathcal{H}_{\mathbb{C}}^{p} \rightarrow \partial \mathcal{H}_{\mathbb{C}}^{q}$ be a $\Gamma$-equivariant measurable map. Then equation (3.1) implies that $\varphi$ satisfies the hypothesis of Theorem 1. Let us show by contradiction that (2) holds. So assume that (1) holds, in particular we may assume that $q=1$. Let us observe that if $I \subset \mathbb{S}^{1}=\partial \mathcal{H}_{\mathbb{C}}^{1}$ is any interval such that $\varphi^{-1}(I)$ is of positive measure, then $\varphi^{-1}(I)$ contains, up to a null set, an open subset of $\partial \mathcal{H}_{\mathbb{C}}^{n}$ : indeed for any $\alpha \neq \beta$ such that $[\alpha, \beta] \in \varphi^{-1}(I)$, the interval

$$
\left\{\zeta \in \partial \mathcal{H}_{\mathbb{C}}^{p}: c_{p}(\alpha, \zeta, \beta)=1\right\} \subset C(\alpha, \beta)
$$

belongs of $\varphi^{-1}(I)$ (up to sets of measure zero), which easily implies the assertion.

Now we claim that $\operatorname{ker} \rho$ is finite and $\Gamma$ is cocompact. To prove the claim, assume that $N:=\operatorname{ker} \rho<\Gamma$ is infinite. Being discrete, its limit set in $\partial \mathcal{H}_{\mathbb{C}}^{p}$ is nonvoid, hence equals $\partial \mathcal{H}_{\mathbb{C}}^{p}$, which implies that $N$ acts minimally on $\partial \mathcal{H}_{\mathbb{C}}^{p}$. Pick any interval $I \subset S^{1}$ such that $\varphi^{-1}(I)$ has positive measure and let $\mathcal{O} \subset$ $\partial \mathcal{H}_{\mathbb{C}}^{p}$ be an open set such that $\mathcal{O}$ is included in $\varphi^{-1}(I)$ up to a set of measure zero. Then $\varphi$ being $N$-invariant, and $N$ acting minimally on $\partial \mathcal{H}_{\mathbb{C}}^{p}$, we have that $\partial \mathcal{H}_{\mathbb{C}}^{p}=\cup_{n \in N} n \mathcal{O}$ is contained in $\varphi^{-1}(I)$, up to measure zero. But since $\varphi$ is not essentially constant, one might find two disjoint intervals $I_{1}$ and $I_{2}$ such that $\varphi^{-1}\left(I_{1}\right)$ and $\varphi^{-1}\left(I_{2}\right)$ are of positive measure and hence of full measure, which is a contradition. This shows that $\operatorname{ker} \rho$ is finite. If $\Gamma$ were not cocompact, then - since $p \geq 2$ - it would contain an integer Heisenberg group which would be sent, almost injectively, into $\mathrm{PU}(1,1)$. Since this is impossible, it follows that $\Gamma$ is cocompact.

Thus $\Gamma$ and $\rho(\Gamma)$ are commensurable, and hence their virtual cohomological dimensions coincide; thus $\rho(\Gamma)$ has virtual cohomological dimension 4 and hence cannot be discrete in $\mathrm{PU}(1,1)$. Being Zariski dense, $\rho(\Gamma)$ is therefore dense in $\mathrm{PU}(1,1)$. Passing to a subgroup of finite index of $\Gamma$, we may in addition assume that $\Gamma$ is torsionfree and $\rho$ is injective. Since the set of elliptic elements is open in $\operatorname{PU}(1,1)$, we may pick $\gamma \neq I d$, with $\rho(\gamma)$ elliptic. Since $\Gamma$ is cocompact and torsionfree, then $\gamma$ is necessarily hyperbolic. Now pick a pair of open intervals $\emptyset \neq I \subset I^{\prime}$ such that the complement of $I^{\prime}$ is of nonvoid interior. Let $\mathcal{O} \subset \partial \mathcal{H}_{\mathbb{C}}^{p}$ be a nonvoid open subset such that $\mathcal{O} \subset \varphi^{-1}(I)$ up to a set of measure zero. Conjugating by an element of $\Gamma$, we may assume that the repulsive fixed point of $\gamma$ is in $\mathcal{O}$. Let now $\left\{n_{k}\right\}_{k \in \mathbb{N}}$ be a divergent sequence of integers such that 
$\lim _{k \rightarrow \infty} \rho(\gamma)^{n_{k}}=I d$ in $\mathrm{PU}(1,1)$; we may assume that $\rho(\gamma)^{n_{k}} I \subset I^{\prime}$ for all $k \geq 1$. Then $\cup_{k \geq 1} \gamma^{n_{k}} \mathcal{O}=\partial \mathcal{H}_{\mathbb{C}}^{p} \backslash\{\xi\}$, where $\xi$ is the attractive fixed point, and hence $\varphi^{-1}\left(I^{\prime}\right)$ equals $\partial \mathcal{H}_{\mathbb{C}}^{p}$ up to a set of measure zero. Since $I^{\prime}$ was arbitrary, this is a contradiction.

\section{REFERENCES}

1. E. Artin, Geometric algebra, Interscience Publishers, New York, 1957.

2. S. B. Bradlow, O. García-Prada, and P. B. Gothen, Surface group representations in $\mathrm{PU}(p, q)$ and Higgs bundles, J. Diff. Geom. 64 (2003), no. 1, 111-170.

3. M. Burger and A. Iozzi, Bounded differential forms, generalized Milnor-Wood inequality and an application to deformation rigidity, Geom. Dedicata 125 (2007), no. 1, 1-23.

4. _ـ A useful formula in bounded cohomology, to appear in "Séminaires et Congrès", http://www.math.ethz.ch/ iozzi/grenoble.ps.

5 . _ـ Bounded cohomology and representation varieties in $\mathrm{PU}(1, n)$, preprint announcement, April 2000.

6. M. Burger, A. Iozzi, F. Labourie, and A. Wienhard, Maximal representations of surface groups: Symplectic Anosov structures, Quarterly Journal of Pure and Applied Mathematics 1 (2005), no. 3, 555-601, Special Issue: In Memory of Armand Borel, Part 2 of 3.

7. M. Burger, A. Iozzi, and A. Wienhard, Surface group representations with maximal Toledo invariant, preprint, http://www.math.ethz.ch/ iozzi/toledo.ps, http://www.arXiv.org/math.DG/0605656.

8. — Surface group representations with maximal Toledo invariant, C. R. Acad. Sci. Paris, Sér. I 336 (2003), 387-390.

9. E. Cartan, Leçons sur la géométrie projective complexe, Gauthier-Villars, Paris, 1931.

10. _ Sur les groupes de la géométrie hypersphérique, Comm. Math. Helv. 4 (1932), 158171.

11. Shiing-shen Chern, Topics in differential geometry, The Institute for Advanced Study, Princeton, N. J., 1951.

12. W. M. Goldman, Discontinuous groups and the Euler class, Thesis, University of California at Berkeley, 1980.

13. _ Characteristic classes and representations of discrete groups of Lie groups, Bull. Amer. Math. Soc. 6 (1982), no. 1, 91-94.

14. W. M. Goldman and J. Millson, Local rigidity of discrete groups acting on complex hyperbolic space, Invent. Math. 88 (1987), 495-520.

15. N. Gusevskii and J. R. Parker, Representations of free Fuchsian groups in complex hyperbolic space, Topology 39 (2000), 33-60.

16. L. Hernández Lamoneda, Maximal representations of surface groups in bounded symmetric domains, Trans. Amer. Math. Soc. 324 (1991), 405-420.

17. Dale Husemoller, Fibre bundles, Graduate Texts in Mathematics, vol. 20, Springer-Verlag, New York, 1994.

18. A. Iozzi, Bounded cohomology, boundary maps, and representations into $\operatorname{Homeo}_{+}\left(S^{1}\right)$ and $S U(1, n)$, Rigidity in Dynamics and Geometry, Cambridge, UK, 2000, Springer Verlag, 2002, pp. 237-260. 
19. V. Koziarz and J. Maubon, Harmonic maps and representations of non-uniform lattices of $\mathrm{PU}(m, 1)$, preprint, http://www.arXiv.org/math.DG/0309193.

20. D. Toledo, Representations of surface groups in complex hyperbolic space, J. Diff. Geom. 29 (1989), no. 1, 125-133.

21. W. T. van Est, Group cohomology and Lie algebra cohomology in Lie groups, I, II, Nederl. Akad. Wetensch. Proc. Series A. 56=Indag. Math. 15 (1953), 484-504.

Marc Burger

FIM, ETH Zentrum, Rämistrasse 101, CH-8092 Zürich, Switzerland

E-mail: burger@math.ethz.ch

\author{
Alessandra Iozzi \\ D-Math, ETH Zentrum, Rämistrasse 101, CH-8092 Zürich, Switzerland \\ E-mail: iozzi@math.ethz.ch
}

